\title{
Rationale, Design, and Baseline Characteristics of a Community- based Comparative Effectiveness Trial to Prevent Type 2 Diabetes in Economically Disadvantaged Adults: The RAPID Study
}

\author{
Ronald T. Ackermann, M.D., M.P.H. ${ }^{a}$, Emily A. Finch, M.A. ${ }^{a}$, Karen K. Schmidt, R.N., \\ M.S.N. ${ }^{b}$, Helena M. Hoen, M.S. ${ }^{c}$, Laura M. Hays, Ph.D. ${ }^{d}$, David G. Marrero, Ph.D. ${ }^{b}$, and \\ Chandan Saha, Ph.D. ${ }^{\mathrm{C}}$ \\ a Department of Medicine, Northwestern University Feinberg School of Medicine, 750 N. Lake \\ Shore Dr., $10^{\text {th }}$ floor, Chicago, IL 60611 \\ b Department of Medicine, Indiana University School of Medicine, $410 \mathrm{~W} 10^{\text {th }} \mathrm{St}$, Suite 3000 , \\ Indianapolis, IN, 46202 \\ c Department of Biostatistics, Indiana University School of Medicine, $410 \mathrm{~W} 10^{\text {th }}$ St, Suite 3000 , \\ Indianapolis, IN 46202 \\ d Indiana University School of Nursing, Indianapolis, 1111 Middle Dr., Indianapolis, IN 46202
}

\section{Abstract}

Reaching out And Preventing Increases in Diabetes (RAPID) is a community-based randomized trial evaluating the comparative costs and effectiveness of a group-based adaption of the DPP lifestyle intervention developed and implemented in partnership with the YMCA.

RAPID enrolled adult primary care patients, with BMI $24 \mathrm{~kg} / \mathrm{m}^{2}$ or higher and abnormal glucose metabolism (HbA1c 5.7-6.9\% or fasting plasma glucose 100-125 mg/dL). 509 participants were enrolled and randomized to one of two groups: standard clinical advice plus free-of-charge access to a group-based adaption of the DPP offered by the Y, versus standard clinical advice alone. Key outcomes for future analysis will include differences in body weight and other cardiovascular risk factors over a 24-month intervention period.

At baseline, RAPID participants had a mean (SD) age of $51 \pm 12.1$ years, weight of $225.1 \pm 56.2$ Ibs, and BMI of $36.9 \pm 8.6 \mathrm{~kg} / \mathrm{m}^{2} .70 .7 \%$ were women, $57.2 \%$ were African American, $35.4 \%$ were non-Hispanic White, and 3.2\% were Hispanic. Mean HbA1c was $6.05 \pm 0.34 \%$.

Additionally, 55.4\% of participants had a baseline systolic blood pressure of $\geq 130 \mathrm{mmHg}, 33.1 \%$

(C) 2013 Elsevier Inc. All rights reserved.

Corresponding Author: Ronald T. Ackermann, M.D., M.P.H.; 750 North Lake Shore Drive, $10^{\text {th }}$ Floor, Chicago, IL, 60611; T: 312-503-6400; r.ackermann@northwestern.edu.

Publisher's Disclaimer: This is a PDF file of an unedited manuscript that has been accepted for publication. As a service to our customers we are providing this early version of the manuscript. The manuscript will undergo copyediting, typesetting, and review of the resulting proof before it is published in its final citable form. Please note that during the production process errors may be discovered which could affect the content, and all legal disclaimers that apply to the journal pertain. 
had a total blood cholesterol exceeding $200 \mathrm{mg} / \mathrm{dl}$, and $74 \%$ reported a household income of < $\$ 25,000$.

The RAPID Study successfully randomized a large cohort of participants with a wide distribution of age, body weight, and race who are at high risk for developing type 2 diabetes.

\section{Keywords}

pre-diabetes; primary prevention; type 2 diabetes mellitus; overweight/obesity; lifestyle intervention; community research

\section{Introduction}

Over 150 million Americans are overweight or obese and at risk for weight-related complications such as cardiovascular disease, stroke, high blood pressure, abnormal blood cholesterol, type 2 diabetes, cancer, osteoarthritis, sleep apnea, depression, and chronic pain. $[1,2]$ To the extent that obesity is a preventable or reversible health condition, many of these complications, along with their associated health implications and costs, could be avoided.

Past research has underscored the striking linkage between type 2 diabetes and obesity,[3] as well as the considerable promise of reducing the burden of diabetes through primary prevention. Several large clinical trials, including the U.S. Diabetes Prevention Program (DPP), demonstrated that resource-intensive lifestyle interventions supporting daily physical activity and modest weight loss can reduce the rate of developing type 2 diabetes by more than half.[4] Despite the promise of such interventions, even modest weight loss is challenging to achieve and maintain,[5] particularly among younger, more obese, minority, and economically disadvantaged persons.[6] Not surprisingly, the cost of offering intensive programs imposes barriers for routinely implementation in real-world settings.[7, 8] One prevailing objective of translational research in diabetes prevention is to adapt evidencebased lifestyle interventions to be more accessible in communities and less costly to implement, without compromising their effectiveness.[9]

Though early research suggests that community-based adaptions of diabetes prevention programs can achieve meaningful weight losses at a lower overall cost, most high risk individuals remain unwilling or unable to pay those costs.[10] The lack of engagement in prevention programs not only threatens the health of individuals but also contributes to recent increases in healthcare expenditures.[11] As a result, there is increasing pressure for commercial health insurance plans and healthcare purchasers, such as employers, Medicaid, and Medicare, to decide whether the benefits of funding prevention programs are compelling enough to warrant their up-front cost and if such coverage is even feasible given the large numbers of persons at risk.

With the need for information to guide decisions by community organizations, health plans, employers, and public officials, we designed the Reaching Out to Prevent Increases in Diabetes (RAPID) Study, a collaborative model for diabetes prevention that links health 
system efforts to identify high risk adults with community-based delivery of a lower cost, group lifestyle intervention offered by trained instructors employed by the YMCA (now known simply as the "Y"). This NIH-funded, community-based comparative effectiveness trial aims to evaluate whether a "Y Model" for translating the DPP lifestyle intervention is capable of reaching large numbers of high risk adults with pre-diabetes and whether that intervention can be cost-effective from the perspective of a health system and society over a 2-year period. The purpose of this manuscript is to describe the design, recruitment, and baseline characteristics of participants in the RAPID Study.

\section{RESEARCH DESIGN AND METHODS}

\section{Study Design and Participants}

RAPID is a two-arm, parallel group, individually randomized intervention trial comparing standard care (including brief counseling) alone versus standard care (including brief counseling) plus referral to the $\mathrm{Y}$ for free-of-charge access to a group-based adaption of the DPP lifestyle intervention (known as PLAN4WARD or simply "P4W"). Study participants are 18 years and older with a BMI $\geq 24 \mathrm{~kg} / \mathrm{m}^{2}$, no prior self-report or clinical diagnosis of type 2 diabetes, and at least one blood test within 6 weeks of enrollment indicating high risk for the development of type 2 diabetes (HbA1c level of 5.7-6.9\% or plasma glucose concentration of $100-125 \mathrm{mg} / \mathrm{dL}$ in the fasting state).

\section{Recruitment Flow}

Nine Indianapolis-area primary care clinics (6 of which were low-income publicly subsidized health centers) received technical assistance from our research team to implement strategies to identify pre-diabetes and offer brief advice to high-risk adults about how to access resources to prevent type 2 diabetes. These clinics were interested in participating because they believed the rapid increases in type 2 diabetes among their patients were making it more difficult to achieve national benchmarks for diabetes care quality. Several of these clinics had previously worked with the research team on pilot studies in this area and were comfortable that the research procedures could be conducted in the clinic without disrupting patient care. Adult patients were informed about diabetes screening opportunities through a variety of strategies including mailed outreach letters, waiting room posters, and risk factor assessments administered by healthcare personnel during routine primary care visits. Patients with blood-tests indicating pre-diabetes were identified through several methods. First, registered dietitians who counseled high risk patients provided study information and referred those interested to research staff. Second, log sheets that captured the results of point-of-care A1c tests were used to identify patients with prior approval of their primary care provider. Third, data managers commissioned by the participating primary care practice group used electronic databases to identify patients who had completed one or more blood tests indicating pre-diabetes in the preceding 12 months. After excluding patients who had evidence of diagnosed diabetes or who were participants of another ongoing research study, the data manager delivered this patient list to ResNet, a local practice-based research network under contract with the practice group. ResNet sought permission from each primary care provider (PCP) to approach all of his/her patients with pre-diabetes. Following PCP approval, each patient was called by phone to explain the study 
and review a short list of preliminary eligibility criteria. Each interested and potentially eligible patient was referred for a face-to-face visit with a Research Assistant (RA) at the clinic and was enrolled if repeat A1c testing confirmed the diagnosis of pre-diabetes, and if he/she provided written informed consent, was able to read English, was not pregnant, was not actively taking a medication known to alter glucose metabolism (e.g. oral steroids or select antipsychotic medications), and did not have a co-morbidity expected to limit lifespan to less than 3 years or that might contraindicate the gradual adoption of light-moderate physical activity (e.g. recent cardiovascular event, severe COPD, advanced arthritis, poorly controlled hypertension). If the patient had not recently seen a Registered Dietitian, the patient was offered an appointment to receive this service. The study protocol was registered in the National Clinical Trials Registry (NCT00656682) and approved by the Indiana University Purdue University Indianapolis IRB.

\section{Metrics and Data Collection}

All participants were asked at the enrollment visit to complete a limited battery of baseline biometric, metabolic, and survey procedures. All measurements were completed by RAs who worked under a business associates agreement within the Indiana University Medical Group and were trained by the Principal Investigator and Research Coordinator. The primary outcome is the between-group difference in body weight over the first 12 months. Body weight was measured using a commercial-grade weight scale, and height was measured using a wall-mounted stadiometer. Secondary outcomes include the percentage of participants who reach weight loss goals of $5 \%$ or more, as well as changes in blood pressure, total and HDL-cholesterol, and hemoglobin A1c (A1c). Blood pressures were assessed as the average of two values measured with an aneroid sphygmomanometer with participants seated and relaxed for at least 5 minutes. If the two readings differed by more than $10 \mathrm{mmHg}$, a third measure was taken and blood pressure was analyzed as the mean of the closest two values. A1c was assessed from a fingerstick capillary blood sample using a DCA 2000/Vantage portable bench-top analyzer.[12, 13] Non-fasting total cholesterol and HDL-C concentrations were measured from capillary blood using an LDX lipid analyzer. $[14,15]$ Because the goal of the study was to demonstrate weight loss effectiveness of the adapted DPP intervention while minimizing the measurement burden on patients and clinicians, the RAPID Study protocol did not replicate DPP clinical trial protocols that also included fasting or timed glucose tolerance testing.

The RAPID Study also collected several survey-based measures that will be used in future analyses, including medical history information and versions of previously developed instruments to assess weight loss and physical activity stages-of-change [16, 17], weight loss and physical activity self-efficacy,[18, 19] dietary and physical activity social support,[20] diabetes risk perceptions, [21, 22] self-report physical activity,[23, 24] and health state utility (using the EuroQol 5-Dimension (EQ-5D) instrument).[25] Additional survey measures included an adapted version of the 17-item National Health Interview Survey (NHIS) Multifactor Screener short-form food frequency questionnaire,[26] with added items to capture changes over time in portion sizes. At follow-up visits, participants were also asked items from the DPP Interval History, DPP Case Report Forms, and self-report healthcare utilization and non-medical care cost survey.[7] 


\section{Randomization and Blinding}

Prior to beginning the study, randomization lists were generated separately for each clinic using SAS, version 9.2 (Cary, N.C.). Lists were created using 1:1 allocation, with blocks of two. The assignment for each sequential study ID was blinded in individually sealed, opaque envelopes kept in a locked filing cabinet accessible only to the RA in each participating primary care study site. Immediately after completing the baseline measures, the RA allocated each participant by opening the next sequentially numbered envelope, which included instructions specific for the treatment condition to which they were randomized. Although this step unblinds the participant to his/her intervention assignment, participants were not told they were being randomized. Instead, all participants were informed during enrollment that the study would provide repeat diabetes risk testing and other risk factor assessments, brief lifestyle counseling at the clinic, and ongoing updates about communitybased lifestyle intervention resources to help them reduce their risk for developing diabetes. These intervention elements were common to both study arms. As a part of the written informed consent, all participants agreed to allow their contact information to be made available to select community organizations that might contact them with information about additional resources. Although contact information was given to the $\mathrm{Y}$ for only those participants assigned to the $\mathrm{P} 4 \mathrm{~W}$ arm, participants remained unaware of the randomization process or that the types of community resource information offered to them may have differed from other participants. We did not disclose the step of randomization because unblinded studies of lifestyle interventions are subject to additional self-report biases and unequal loss to follow up if participants are aware they are receiving a less intensive treatment that they might perceive to be more beneficial (i.e. controls). Control participants in this study were not aware that some participants were offered a more intensive lifestyle intervention program at the Y. This form of "behind the scenes" randomization is common in pragmatic effectiveness trials where an intervention has established efficacy and no more than minimal risk to subjects, meaning that the step of randomizing adds no additional risks and simply allows the intervention (which cannot be offered to all patients) to be offered fairly (i.e. by coin flip), while also creating an unbiased control group who are guaranteed additional standard information, education, and follow-up for their condition.

\section{Interventions}

At the close of the first study visit, all participants received a study welcome packet and were encouraged to utilize the resources provided to assist them with goals toward diabetes prevention. Participants in both treatment arms (standard care; P4W) received information regarding community resources and self-help materials from the National Diabetes Education Program (NDEP)[27] to support goal-setting and the adoption of moderate physical activities and dietary changes to achieve modest weight loss. These community resources and self-help materials were provided in a written format by both study RAs and RDs; if the patient expressed interest, the study staff would verbally review the written resource materials with the patient and help the patient focus on particular resources most appropriate for him/her. This information was revisited at each subsequent study visit. RAs encouraged and assisted participants in both arms to arrange a visit with a registered dietitian, already employed by the practice, to obtain further assistance in developing an action plan for dietary changes and weight loss. 
In addition to this basic information offered to all participants, those in the P4W arm were also given a flier with information about how to access P4W free-of-charge through a nearby Y. Within 24 business hours of enrollment, a RAPID Study staff member called a dedicated Y-based P4W intervention coordinator and provided the name and contact information for any new participants assigned to the $\mathrm{P} 4 \mathrm{~W}$ arm. The $\mathrm{Y}$ coordinator then initiated a series of attempts to enroll each participant in an upcoming P4W group session, at a time and location that were most convenient for the participant. P4W enrollment was actively encouraged but not required for ongoing participation in the study.

\section{P4W Intervention Goals and Description}

The P4W intervention was based on the DPP approach and involves a structure of goalsetting, self-monitoring, and participant-centered problem solving to achieve modest weight loss (5-7\% reduction from baseline) through a combination of moderate physical activity (150 minutes per week equivalent to walking) and lower dietary fat and total calorie consumption.[28] In a prior study, the group-based P4W intervention was delivered by the Y with fidelity to the DPP model and achieved $6 \%$ mean weight loss after 6 and 12 months and 4.8\% (SE 1.4\%) mean weight loss after 28 months among high risk community volunteers. $[29,30]$ The $Y$ has remained persistently committed to offering this intervention because it aligns with organizational values for health promotion, as well as growing interests to partner with healthcare, public health, and other stakeholders to preserve and improve population health.

P4W participants were assembled by the Y P4W coordinator into groups of 8-12 persons who could meet at a mutually agreeable time and location. Based on referral volumes and personal preference, some study participants were able to begin participating in $\mathrm{P} 4 \mathrm{~W}$ immediately, while others did not begin for as long as 6 months after study enrollment. Because the goal of this effectiveness trial was to evaluate the overall treatment effect of offering the DPP under "real-world" conditions, participants were not given additional incentives to participate or excluded from the trial for their lack of P4W participation, and data collection still proceeded on schedule (i.e. 6 and 12 months after baseline data collection) for all participants. P4W intervention locations included $\mathrm{Y}$ facilities and non-Y locations in the community, including delivery in meeting spaces within participating primary care clinics.

P4W involves a "core curriculum" comprised of 16 classroom-style behavioral counseling meetings, each lasting about 60 to 90 minutes and delivered over a period of 16 to 20 weeks. [31] Following completion of the core curriculum visits, participants were offered monthly, 60-minute maintenance lessons for the full duration of the trial.[30, 32] "Toolkit" incentives used in the DPP clinical trial to increase adherence to intervention protocols were not used in RAPID, but the $\mathrm{Y}$ did offer many participants (who were largely not $\mathrm{Y}$ members) limited guest-access to the $\mathrm{Y}$, as well as other tools such as a step counter, measuring cups, spoons, scales, fat and calorie tracking tools, recipe guides, and other resources derived from DPP and previous $\mathrm{Y}$ programs. 


\section{P4W Instructor Training}

The P4W intervention was delivered by $31 \mathrm{Y}$ personnel who underwent a 2-day training and recognition program.[33] Intervention locations included 6 different $Y$ facilities associated with the YMCA of Greater Indianapolis. P4W instructor candidates were not required to have a specific academic degree or minimum level of prior training but were selected by $\mathrm{Y}$ administrators based on interest in diabetes prevention and capability to manage groups of wellness program participants. Instructors were typically part-time employees who had a mix of past training and experience with health education, coaching, and wellness programming. Training events included an introduction to diabetes, an overview of the DPP lesson structure, skill-building in the area of managing group dynamics, and a mix of roleplaying and feedback from mock lessons.

Following the training, instructors were supported through ongoing direct access to one another (quarterly peer-to-peer problem solving meetings), as well as to master trainers, project staff, and clinicians on the research team. The Y P4W coordinator completed the training and worked within the $\mathrm{Y}$ to develop procedures for direct observation of each instructor for quality assurance and intervention fidelity [33]. Throughout the trial, refresher training was offered annually and required attendance at a day-long, small group workshop, as well as the completion of written tests that revisited and assessed core competencies of the DPP intervention. All Y group instructors and the P4W coordinator were paid at rates typical for similar Y staff.

\section{Statistical Analysis and Planned Analyses:}

Analyses for this manuscript involved descriptive statistics summarizing the timing and process of recruitment approaches, as well as the baseline characteristics of the randomized study cohort. Future manuscripts will report on changes in body weight, other biometric, metabolic, and self-report outcomes, and costs. The principal analysis will focus on differences in body weight between randomized treatment arms following the intent to treat principal. However, because this is an effectiveness trial, we do not expect that all study participants who are offered $\mathrm{P} 4 \mathrm{~W}$ will attend the program. Thus, we will also estimate separately the probability of attendance when offered P4W, and the effect of P4W when it is received. Although such comparisons of self-selected subgroups can introduce various forms of selection bias we will use random treatment assignment as an instrumental variable to minimize that bias.[34-36]

The targeted sample size of 508 participants was expected to provide more than $80 \%$ power at the $\mathrm{a}=0.05$ significance level to detect an absolute between-group mean difference in the percent weight loss at 12 months as low as $2.2 \%$ (SD 4.3\%) among all randomized participants, as well as within the African American subgroup. This calculation assumes enrollment a minimum of $40 \%$ African Americans, 9 participating primary care clinics, an intra-cluster correlation of $2 \%$ or less for changes in weight within each clinic, and attrition not exceeding $15 \%$ at 12-months. These analyses will further conserve statistical power by using repeated measures ANOVA estimation. 


\section{RESULTS}

\section{Recruitment Channels}

Over the study's 29-month recruitment period, the 9 participating primary care clinics

logged about 115,300 adult patient visits per year. The demographic characteristics of those patients varied by clinic and are presented in Table 1 .

Using clinical database queries, 12,787 patients were identified during the 29-month recruitment period as having completed a fasting glucose test during routine clinical care (Table 1). Results of these tests yielded 3,064 (23.9\%) patients who were in the high risk range (i.e. FPG 100-125 mg/dL). In parallel with RAPID Study recruitment, 6 of the 9 participating clinic sites implemented standing order protocols and practice incentives to increase the completion of annual A1c testing among adults with risk factors for diabetes. Through A1c testing, an additional 5,217 patients completed diabetes screening, yielding 2,137 added patients with high risk A1c test results. The success of attempts to recruit these high risk patients varied considerably by clinic, with more successful recruitment in clinics that performed more diabetes tests. In the year prior to the study, total numbers of diabetes tests performed varied among clinics, but testing increased most during the study in clinics that implemented targeted A1c testing programs. In those 6 clinics, the percentage of high risk patients in each clinic who underwent a screening blood test increased by $124 \%$ between the period 1 year before and 1 year after implementing A1c testing programs. Testing in the remaining 3 clinics increased only $6 \%$ over the same period. An overview of the success of strategic protocol changes to enhance recruitment is depicted in Figure 1.

Over the entire 29 months of recruitment, 509 participants were enrolled and randomized from among 3,064 patients who were identified with a high-risk glucose test and whose physician provided approval for recruitment. The entire recruitment flow is summarized in Figure 2. By assuming that the eligibility rate would have been the same among patients who refused screening or who could not be contacted, the 509 enrolled participants would represent $36 \%$ of all patients estimated as eligible in participating practices. The largest challenge in the recruitment flow stemmed from being unsuccessful to secure approval from some PCPs to approach their potentially eligible patients (unable to secure approval for $41 \%$ of patients with high glucose). The most common reason for this was lack of physician responsiveness, rather than overtly declining that patients be approached. Although our protocol did not enable us to collect detailed information for each reason an eligible patient opted out, inaccurate contact information was a common explanation for not reaching patients by phone. In addition, a large number of patients who were reached expressed that they had limited transportation or were too busy and had no time to participate in research.

Common reasons for ineligibility ( $\mathrm{n}=849$ ) among the 1,358 participants who were screened for the study included about $30 \%$ who reported chest pain, heart problems, breathing problems, arthritis or other reasons for not being able to increase their physical activity even gradually; 24\% who reported being unable to read written English; 13\% who were taking an anti-psychotic or oral steroid medication that could interfere with blood glucose; $11 \%$ who reported being diagnosed with diabetes or already taking a diabetes medication; $9 \%$ who 
reported having a more recent blood glucose test that was normal; and 6\% who had a normal body mass index.

\section{Baseline Characteristics of the Randomized Cohort}

The characteristics of randomized participants are shown in Table 2. The overall mean $( \pm$ $\mathrm{SD})$ age at randomization was $51.0 \pm 12.1$ years. More than $70 \%$ of participants were women and 57\% were African-American. Seventy-four percent of participants reported a household income of $\langle \$ 25,000$, with a mean household size of 2.7 persons. The mean weight $\left( \pm \mathrm{SD}\right.$ ) was $225.1 \pm 56.2 \mathrm{Ibs}$, and mean BMI was $36.9 \pm 8.6 \mathrm{~kg} / \mathrm{m}^{2}$.

About $58 \%$ of participants reported a sibling or parent with diagnosed diabetes. Clinical cardiovascular risk factors were modestly elevated, reflecting the underlying high metabolic risk of participants but also their recruitment from primary health care settings, where blood pressure and antilipidemic medications could be received. Mean A1c was 6.1\%. Mean systolic blood pressure was $132 \mathrm{mmHg}$, and was $130 \mathrm{mmHg}$ or higher in $55.4 \%$ of participants and $140 \mathrm{mmHg}$ or higher in $33.3 \%$ of participants. $61 \%$ reported taking at least one blood pressure medication. Mean HDL-C was $42.7 \mathrm{mg} / \mathrm{dL}$ and was below $40 \mathrm{mg} / \mathrm{dL}$ for approximately $45.4 \%$ of participants. Mean total cholesterol was $185.8 \mathrm{mg} / \mathrm{dL}$ and exceeded $200 \mathrm{mg} / \mathrm{dL}$ in $33.1 \%$ of participants.

\section{CONCLUSIONS}

The Reaching out And Preventing Increases in Diabetes (RAPID) Study successfully recruited and randomized 509 adults with abnormal glucose metabolism, elevated BMI, and increased cardiovascular risk factor levels. Participants reflect the underlying population of a diverse mix of 9 primary care clinic recruitment sites. Successful recruitment took considerable time despite the underlying high prevalence of pre-diabetes in adults. A major challenge for recruitment was that clinical practice recommendations for targeted testing of adults have not been routinely implemented.[37, 38] Interestingly, prior to a push for A1c testing by 6 clinics, most fasting glucose tests were identified in conjunction with recent fasting lipid tests, suggesting that some providers recognized the related risks of dysglycemia and hyperlipidemia, and that the goals for treating hyperlipidemia, much like high blood pressure, are impacted by the co-existence of a diabetes diagnosis.[39, 40] This does not suggest, however, that glucose testing is being performed to identify and initiate interventions for pre-diabetes.

Midway through the recruitment phase of the RAPID Study, 6 clinics implemented targeted testing of high risk individuals using hemoglobin A1c. After that change, overall testing among high risk adults increased $124 \%$ over the prior year. In parallel with that increase in testing, recruitment into RAPID increased almost immediately from 4 participants per month to almost 36 participants per month (9-fold increase, depicted in Figure 1). This observation underscores the importance of performing glucose testing as a necessary first step in the management of pre-diabetes. If individuals and their PCPs remain unaware of diabetes risk status, they will remain ill-prepared to consider interventions that could improve health and prevent or delay the development of type 2 diabetes.

Contemp Clin Trials. Author manuscript; available in PMC 2015 March 25. 
It is relevant to compare the characteristics of RAPID Study participants with those of participants in the Diabetes Prevention Program (DPP) clinical trial. Compared with DPP, RAPID participants were generally of similar age (51 years in both studies) and sex (71\% women vs. $68 \%$ in DPP), but had a higher BMI $\left(36.9 \mathrm{~kg} / \mathrm{m}^{2}\right.$ vs. $34.0 \mathrm{~kg} / \mathrm{m}^{2}$ in DPP) and were more likely to report African American race (57\% vs. $20 \%$ in DPP) and having a household income below $\$ 25,000$ (74\% vs. about $20 \%$ in DPP).[41] Compared to DPP, mean A1c at baseline was also slightly higher (6.1\% vs. 5.9\% in DPP), SBP was higher (132 $\mathrm{mmHg}$ vs. $124 \mathrm{mmHg}$ in DPP), mean total cholesterol was lower $(186 \mathrm{mg} / \mathrm{dL}$ vs. $203 \mathrm{mg} / \mathrm{dL}$ in DPP), and mean HDL-C was comparable (42.7 mg/dL vs. $43.9 \mathrm{mg} / \mathrm{dL}$ in DPP).[4, 41, 42]

Past lifestyle intervention trials such as the DPP have observed lower weight loss success among African Americans, women, and those who are younger, more obese, or of low socio-economic position.[6] Thus, the RAPID Study is uniquely positioned to evaluate the capacity of the $\mathrm{Y}$ to deliver the DPP in a way that achieves clinically meaningful weight losses and improved cardiovascular risk factors among adults who have proved most difficult to reach using structured diet and physical activity programs. If successful, our study will advance our understanding for how to reduce inequalities in diabetes risk and how to provide evidence-based interventions in a way that reaches broad segments of high risk adults.

Another recent trial, the Healthy Living Partnerships to Prevent Diabetes (HELP-PD) Study, also involved the translation and implementation of the DPP by trained community health workers. HELP-PD found an adjusted mean difference of $5.7 \%$ greater weight loss among intensive lifestyle intervention participants compared with brief education and support.[43] HELP-PD was a well-conducted randomized trial that reported high attendance (79\%) in intensive intervention sessions over the first year and data collection completed by more than $90 \%$ of subjects at 12 months. Compared with the RAPID cohort, HELP-PD participants were more often men ( $42 \%$ vs. $29 \%$ in RAPID), non-Hispanic White (74\% vs. $35 \%$ in RAPID), older (mean age 58 years vs. 51 years in RAPID), less obese (mean BMI $32.8 \mathrm{~kg} / \mathrm{m}^{2}$ vs. $36.9 \mathrm{~kg} / \mathrm{m} 2$ in RAPID), and likely more affluent ( $80 \%$ with more than a high school education in HELP-PD vs. only $26 \%$ reporting household income $>\$ 25,000$ in RAPID).[44] HELP-PD recruited participants using procedures designed to maximize data collection and intervention participation more than to enroll a study population reflecting a "typical" person in the community with pre-diabetes.[45] Thus, again, RAPID is unique in its enrollment of a more underserved, minority, and obese study sample, and will advance what we can learn about both the reach and effectiveness of offering an adapted version of the DPP to a particularly high need population.

Because RAPID has been designed uniquely to study the effectiveness of a group-based adaption of the DPP among a largely underserved and vulnerable, high-risk population, we anticipate that we may observe lower levels of participation in lifestyle intervention lessons and possibly more modest, yet still meaningful changes in body weight. Importantly, we also expect that intervention costs will be lower and that improvements in body weight and other cardiometabolic risk factors will increase among those persons who attend more intervention visits. In addition to assessing the overall cost-effectiveness of this group adaption of the DPP intervention, the RAPID study is designed to provide valid estimates of 
the intervention's effectiveness among the subgroup of high risk primary care patients who attend the program. It is our hope that this information will advance research as well as guide organizational and public policy decisions regarding how best to implement and sustain new activities to address the growing burden of type 2 diabetes in an equitable and cost-effective way.

\section{Acknowledgements}

Support for this study was provided by the National Institute of Diabetes and Digestive and Kidney Diseases (R18 DK079855) and the Robert Wood Johnson Foundation (\#57398). R.T. Ackermann is also supported by the Northwestern University Clinical and Translational Sciences Institute (UL1RR025741). We recognize the DPP Study Group and specifically the Lifestyle Resource Core at the University of Pittsburgh, who led the development of the initial DPP intervention. We would also like to recognize the support and participation of the YMCA of Greater Indianapolis, the providers and staff of IU Medical Group - Primary Care, the Regenstrief Institute, Inc., and all RAPID Study participants.

\section{References}

1. Flegal KM, Carroll MD, Ogden CL, Curtin LR. Prevalence and trends in obesity among US adults, 1999-2008. JAMA. 2010; 303:235-41. [PubMed: 20071471]

2. US Census Bureau. Population Estimates. U.S. Census Bureau, Population Division; Washington, DC: 2009. available at: http://www.census.gov/popest/national/asrh/

3. Gregg EW, Cheng YJ, Cadwell BL, Imperatore G, Williams DE, Flegal KM, et al. Secular trends in cardiovascular disease risk factors according to body mass index in US adults. JAMA. 2005; 293:1868-74. [PubMed: 15840861]

4. Diabetes Prevention Program Research Group. Reduction in the incidence of type 2 diabetes with lifestyle intervention or metformin. N Engl J Med. 2002; 346:393-403. [PubMed: 11832527]

5. McTigue KM, Harris R, Hemphill B, Lux L, Sutton S, Bunton AJ, et al. Screening and interventions for obesity in adults: summary of the evidence for the U.S. Preventive Services Task Force. Ann Intern Med. 2003; 139:933-49. [PubMed: 14644897]

6. Wing RR, Hamman RF, Bray GA, Delahanty L, Edelstein SL, Hill JO, et al. Achieving weight and activity goals among diabetes prevention program lifestyle participants. Obes Res. 2004; 12:142634. [PubMed: 15483207]

7. Herman WH, Brandle M, Zhang P, Williamson DF, Matulik MJ, Ratner RE, et al. Costs associated with the primary prevention of type 2 diabetes mellitus in the diabetes prevention program. Diabetes Care. 2003; 26:36-47. [PubMed: 12502656]

8. Garfield SA, Malozowski S, Chin MH, Venkat Narayan KM, Glasgow RE, Green LW, et al. Considerations for diabetes translational research in real-world settings. Diabetes Care. 2003; 26:2670-4. [PubMed: 12941736]

9. Ali MK, Echouffo-Tcheugui J, Williamson DF. How effective were lifestyle interventions in realworld settings that were modeled on the Diabetes Prevention Program? Health Aff (Millwood). 2012; 31:67-75. [PubMed: 22232096]

10. Johnson FR, Manjunath R, Mansfield CA, Clayton LJ, Hoerger TJ, Zhang P. High-risk individuals' willingness to pay for diabetes risk-reduction programs. Diabetes Care. 2006; 29:1351-6. [PubMed: 16732020]

11. Thorpe KE, Howard DH. The rise in spending among Medicare beneficiaries: the role of chronic disease prevalence and changes in treatment intensity. Health Aff (Millwood). 2006; 25:w378-88. [PubMed: 16926180]

12. John WG, Edwards R, Price CP. Laboratory evaluation of the DCA 2000 clinic HbA1c immunoassay analyser. Ann Clin Biochem. 1994; 31(Pt 4):367-70. [PubMed: 7979104]

13. Tamborlane WV, Kollman C, Steffes MW, Ruedy KJ, Dongyuan X, Beck RW, et al. Comparison of fingerstick hemoglobin A1c levels assayed by DCA 2000 with the DCCT/EDIC central laboratory assay: results of a Diabetes Research in Children Network (DirecNet) Study. Pediatr Diabetes. 2005; 6:13-6. [PubMed: 15787896] 
14. Santee J. Accuracy and precision of the Cholestech LDX System in monitoring blood lipid levels. Am J Health Syst Pharm. 2002; 59:1774-9. [PubMed: 12298117]

15. Bard RL, Kaminsky LA, Whaley MH, Zajakowski S. Evaluation of lipid profile measurements obtained from the Cholestech L.D.X analyzer. J Cardiopulm Rehabil. 1997; 17:413-8. [PubMed: 9421763]

16. Hawkins DS, Hornsby PP, Schorling JB. Stages of change and weight loss among rural African American women. Obes Res. 2001; 9:59-67. [PubMed: 11346668]

17. Sarkin JA, Johnson SS, Prochaska JO, Prochaska JM. Applying the transtheoretical model to regular moderate exercise in an overweight population: validation of a stages of change measure. Prev Med. 2001; 33:462-9. [PubMed: 11676588]

18. Clark MM, Abrams DB, Niaura RS, Eaton CA, Rossi JS. Self-efficacy in weight management. J Consult Clin Psychol. 1991; 59:739-44. [PubMed: 1955608]

19. Sallis JF, Pinski RB, Grossman RM, Patterson TL, Nader PR. The development of self-efficacy scales for health-related diet and exercise behaviors. Health Educ Res. 1988; 3:283-92.

20. Sallis JF, Grossman RM, Pinski RB, Patterson TL, Nader PR. The development of scales to measure social support for diet and exercise behaviors. Prev Med. 1987; 16:825-36. [PubMed: 3432232]

21. Walker EA, Caban A, Schechter CB, Basch CE, Blanco E, DeWitt T, et al. Measuring comparative risk perceptions in an urban minority population: the risk perception survey for diabetes. Diabetes Educ. 2007; 33:103-10. [PubMed: 17272796]

22. Walker EA, Mertz CK, Kalten MR, Flynn J. Risk perception for developing diabetes: comparative risk judgments of physicians. Diabetes Care. 2003; 26:2543-8. [PubMed: 12941716]

23. Blair SN, Haskell WL, Ho P, Paffenbarger RS Jr. Vranizan KM, Farquhar JW, et al. Assessment of habitual physical activity by a seven-day recall in a community survey and controlled experiments. Am J Epidemiol. 1985; 122:794-804. [PubMed: 3876763]

24. Pereira MA, FitzerGerald SJ, Gregg EW, Joswiak ML, Ryan WJ, Suminski RR, et al. A collection of Physical Activity Questionnaires for health-related research. Med Sci Sports Exerc. 1997; 29:S1-205. [PubMed: 9243481]

25. Shaw JW, Johnson JA, Coons SJ. US valuation of the EQ-5D health states: development and testing of the D1 valuation model. Med Care. 2005; 43:203-20. [PubMed: 15725977]

26. Thompson FE, Midthune D, Subar AF, McNeel T, Berrigan D, Kipnis V. Dietary intake estimates in the National Health Interview Survey, 2000: methodology, results, and interpretation. J Am Diet Assoc. 2005; 105:352-63. quiz 487. [PubMed: 15746822]

27. National Diabetes Education Program: Diabetes Prevention. National Institutes of Health, the Centers for Disease Control and Prevention, Department of Health and Human Services; Bethesda, MD: 2010. available at: http://www.ndep.nih.gov/publications/

28. Ackermann RT, Marrero DG. Adapting the Diabetes Prevention Program lifestyle intervention for delivery in the community: the YMCA model. Diabetes Educ. 2007; 33:69, 74-5, 7-8. [PubMed: 17272794]

29. Ackermann RT, Finch EA, Brizendine E, Zhou H, Marrero DG. Translating the Diabetes Prevention Program into the community. The DEPLOY Pilot Study. Am J Prev Med. 2008; 35:357-63. [PubMed: 18779029]

30. Ackermann RT, Finch EA, Caffrey HM, Lipscomb ER, Hays LM, Saha C. Long-term effects of a community-based lifestyle intervention to prevent type 2 diabetes: the DEPLOY extension pilot study. Chronic Illn. 2011

31. DPP Research Group on behalf of the University of Pittsburgh. DPP Lifestyle Materials for Sessions 1-16 -- Lifestyle Coach Materials and Optional Participant Handouts. 2002. available at: http://www.bsc.gwu.edu/dpp/lifestyle/dpp_dcor.html

32. DPP Research Group on behalf of the University of Pittsburgh. DPP Lifestyle Materials for Beyond Session 16 -- Lifestyle Coach Materials and Participant Handouts. 2002. available at: http://www.bsc.gwu.edu/dpp/lifestyle/dpp_acor.html

33. Finch EA, Kelly MS, Marrero DG, Ackermann RT. Training YMCA wellness instructors to deliver an adapted version of the Diabetes Prevention Program lifestyle intervention. Diabetes Educ. 2009; 35:224-8, 32. [PubMed: 19321808] 
34. Sussman JB, Hayward RA. An IV for the RCT: using instrumental variables to adjust for treatment contamination in randomised controlled trials. BMJ. 2010; 340:c2073. [PubMed: 20442226]

35. Zhou XH, Li SM. ITT analysis of randomized encouragement design studies with missing data. Statistics in medicine. 2006; 25:2737-61. [PubMed: 16287216]

36. Schoenbaum M, Unutzer J, McCaffrey D, Duan N, Sherbourne C, Wells KB. The effects of primary care depression treatment on patients' clinical status and employment. Health Serv Res. 2002; 37:1145-58. [PubMed: 12479490]

37. Ealovega MW, Tabaei BP, Brandle M, Burke R, Herman WH. Opportunistic screening for diabetes in routine clinical practice. Diabetes Care. 2004; 27:9-12. [PubMed: 14693958]

38. Geiss LS, James C, Gregg EW, Albright A, Williamson DF, Cowie CC. Diabetes risk reduction behaviors among U.S. adults with prediabetes. Am J Prev Med. 2010; 38:403-9. [PubMed: 20307809]

39. The Seventh Report of the Joint National Committee on Prevention, Detection, Evaluation, and Treatment of High Blood Pressure. National Heart, Lung, and Blood Institute. U.S. Department of Health and Human Services; Bethesda, MD: 2004. p. i-88.

40. Grundy SM, Cleeman JI, Merz CN, Brewer HB Jr. Clark LT, Hunninghake DB, et al. Implications of recent clinical trials for the National Cholesterol Education Program Adult Treatment Panel III guidelines. Circulation. 2004; 110:227-39. [PubMed: 15249516]

41. Diabetes Prevention Program Research Group. The Diabetes Prevention Program: baseline characteristics of the randomized cohort. The Diabetes Prevention Program Research Group. Diabetes Care. 2000; 23:1619-29. [PubMed: 11092283]

42. Ratner R, Goldberg R, Haffner S, Marcovina S, Orchard T, Fowler S, et al. Impact of intensive lifestyle and metformin therapy on cardiovascular disease risk factors in the diabetes prevention program. Diabetes Care. 2005; 28:888-94. [PubMed: 15793191]

43. Katula JA, Vitolins MZ, Rosenberger EL, Blackwell CS, Morgan TM, Lawlor MS, et al. One-year results of a community-based translation of the Diabetes Prevention Program: Healthy-Living Partnerships to Prevent Diabetes (HELP PD) Project. Diabetes Care. 2011; 34:1451-7. [PubMed: 21593290]

44. Blackwell CS, Foster KA, Isom S, Katula JA, Vitolins MZ, Rosenberger EL, et al. Healthy Living Partnerships to Prevent Diabetes: recruitment and baseline characteristics. Contemp Clin Trials. 2011; 32:40-9. [PubMed: 20974289]

45. Katula JA, Vitolins MZ, Rosenberger EL, Blackwell C, Espeland MA, Lawlor MS, et al. Healthy Living Partnerships to Prevent Diabetes (HELP PD): design and methods. Contemp Clin Trials. 2010; 31:71-81. [PubMed: 19758580] 


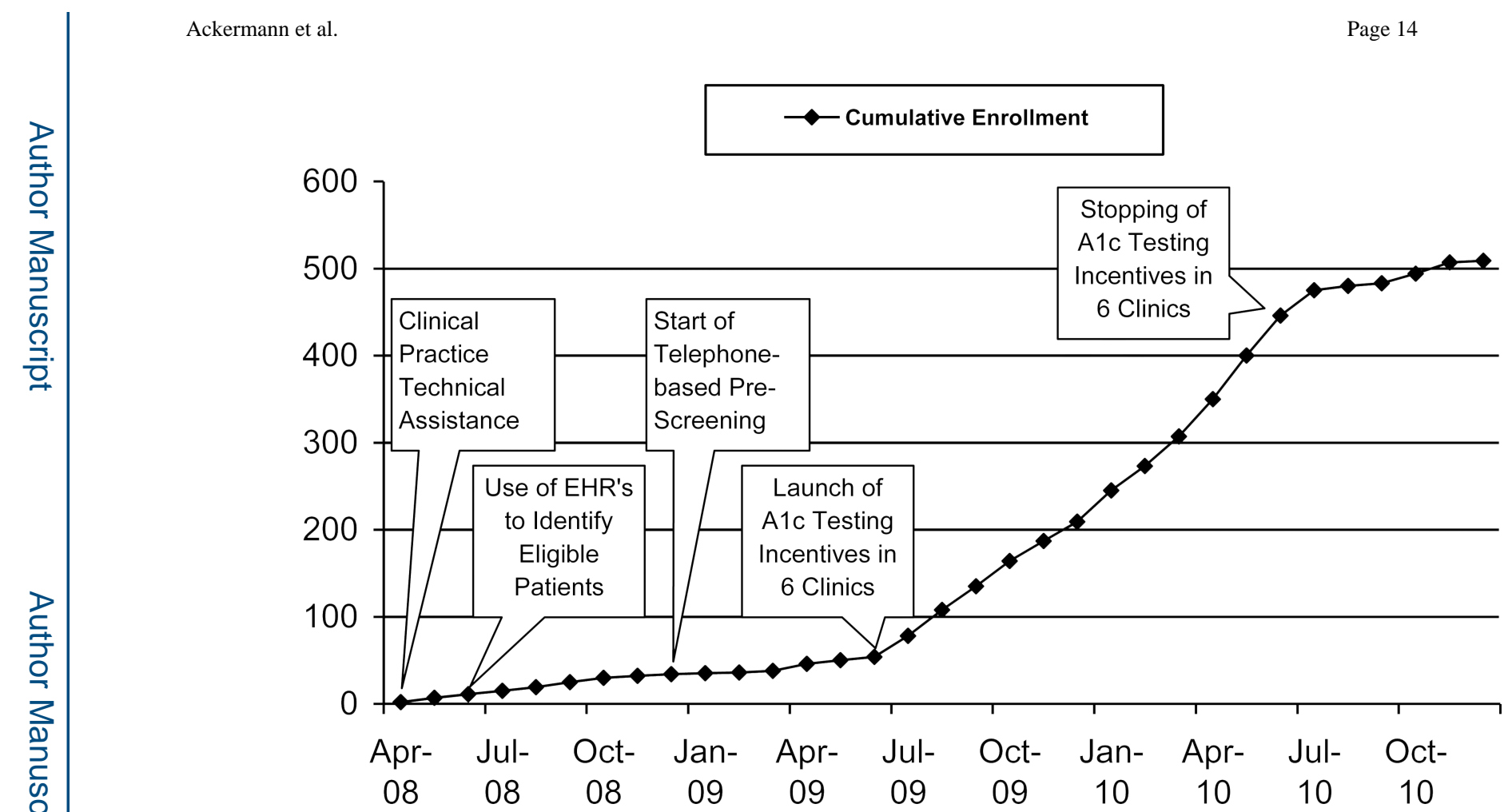

Figure 1.

Relationship of Clinical and Protocol Changes to Recruitment Success Callout boxes describe significant protocol changes implemented to increase recruitment success; the line represents cumulative enrollment; increases in the slope of the line following a protocol change indicate more rapid recruitment success 


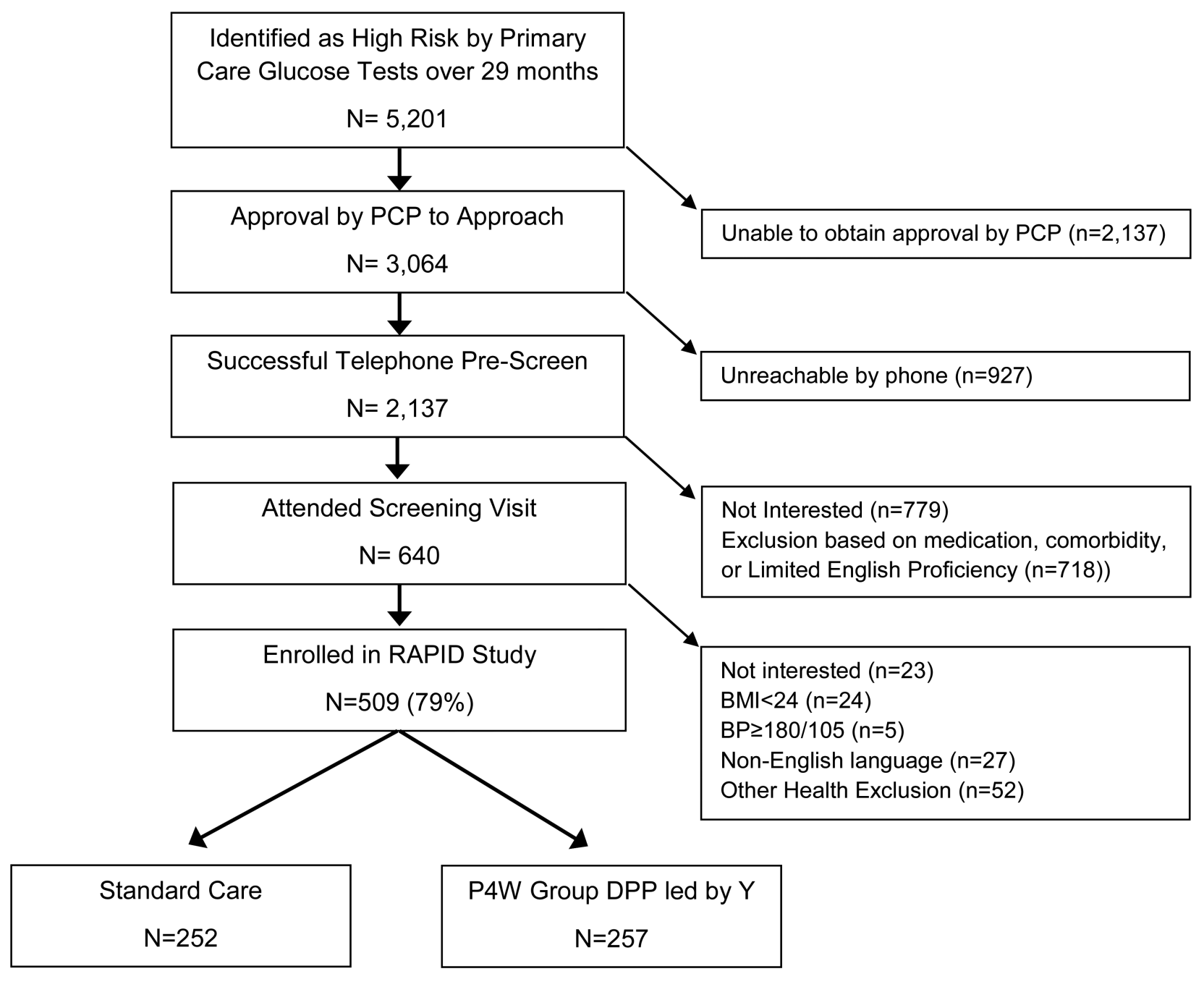

Figure 2. Trial Flow

Boxes and arrows indicate the flow of potentially eligible patients as deemed approachable, eligible, enrolled, and randomized; side arrows provide reasons for non-approach, ineligibility, and non-enrollment 


\section{Table 1}

\section{Characteristics of All Adult Patients of Participating Primary Care Clinics}

\begin{tabular}{|c|c|}
\hline Characteristic & Total \\
\hline Total adults with visits in 2010 & 43,475 \\
\hline$\%$ Female & $71.7 \%$ \\
\hline \multicolumn{2}{|l|}{$\underline{\text { Age }}$} \\
\hline $18-64$ & $38,369(88.3 \%)$ \\
\hline $65+$ & $5,106(11.7 \%)$ \\
\hline \multicolumn{2}{|l|}{$\underline{\text { Race/Ethnicity }}{ }^{*}$} \\
\hline African American & $18,883(43.4 \%)$ \\
\hline Non-Hispanic White & $18,043(41.5 \%)$ \\
\hline Hispanic & $2,278(5.2 \%)$ \\
\hline Other or Unknown Race & $3,930(9.0 \%)$ \\
\hline Diabetes Screening over 29 Months $^{\dagger}$ & 18,004 \\
\hline Pre-diabetes ${ }^{*}$ identified over 29 months & 5,201 \\
\hline \multicolumn{2}{|c|}{ * Based on self-reported race and ethnicity data collected by clinics } \\
\hline
\end{tabular}




\section{Table 2}

\section{Baseline Characteristics of the Randomized Cohort}

\begin{tabular}{|c|c|c|c|}
\hline Characteristic & Overall & Standard Care & P4W Intervention led by $Y$ \\
\hline $\mathrm{N}$ & 509 & 252 & 257 \\
\hline Age (years) & $51.0(12.1)$ & $51.2(12.0)$ & $50.8(12.2)$ \\
\hline Male & $149(29.3)$ & $79(31.3)$ & $70(27.2)$ \\
\hline Female & $360(70.7)$ & $173(68.7)$ & $187(72.8)$ \\
\hline African American & $290(57.0)$ & $143(56.8)$ & $147(57.2)$ \\
\hline Non-Hispanic White & $178(35.0)$ & $87(34.5)$ & $91(35.4)$ \\
\hline Hispanic or Latino & $16(3.1)$ & $4(1.6)$ & $12(4.7)$ \\
\hline Other or Multi-Race & $25(4.9)$ & $18(7.1)$ & $7(2.7)$ \\
\hline BMI $\left(\mathrm{kg} / \mathrm{m}^{2}\right)$ & $36.9(8.6)$ & $36.53(8.36)$ & $37.3(8.8)$ \\
\hline Weight (lbs) & $225.1(55.9)$ & $223.8(55.9)$ & $226.6(56.5)$ \\
\hline Alc & $6.1(0.3)$ & $6.0(0.3)$ & $6.1(0.3)$ \\
\hline Systolic Blood Pressure (mmHg) & $132.2(14.6)$ & $130.9(13.8)$ & $133.5(15.2)$ \\
\hline Diastolic Blood Pressure (mmHg) & $80.3(9.3)$ & $80.0(9.3)$ & $80.5(9.2)$ \\
\hline Total Cholesterol (mg/dl) & $185.8(40.0)$ & $184.5(39.7)$ & $187.0(40.3)$ \\
\hline HDL-C (mg/dl) & $42.7(14.8)$ & $42.1(14.2)$ & $43.2(15.4)$ \\
\hline Household Income & $\mathrm{n}=422$ & $\mathrm{n}=205$ & $\mathrm{n}=217$ \\
\hline$<\$ 10,000$ & $137(32.5)$ & $68(33.2)$ & $69(31.8)$ \\
\hline$\$ 10 \mathrm{~K}-\$ 14.99 \mathrm{~K}$ & $83(19.7)$ & $36(17.6)$ & 47 (21.7) \\
\hline$\$ 15 \mathrm{~K}-\$ 24.99 \mathrm{~K}$ & $92(21.8)$ & $44(21.5)$ & $48(22.1)$ \\
\hline$\$ 25 \mathrm{~K}-\$ 34.99 \mathrm{~K}$ & $39(9.2)$ & $17(8.3)$ & $22(10.1)$ \\
\hline$\$ 35 \mathrm{~K}-\$ 49.99 \mathrm{~K}$ & $32(7.6)$ & $20(9.8)$ & $12(5.5)$ \\
\hline$\$ 50 \mathrm{~K}-\$ 74.99 \mathrm{~K}$ & $19(4.5)$ & $9(4.4)$ & $10(4.6)$ \\
\hline$\geq \$ 75,000$ & $20(4.7)$ & $11(5.4)$ & $9(4.2)$ \\
\hline \multirow[t]{2}{*}{ Family History Type 2 Diabetes } & $\mathrm{n}=503$ & $\mathrm{n}=249$ & $\mathrm{n}=254$ \\
\hline & $290(57.7)$ & $143(57.4)$ & $147(57.9)$ \\
\hline
\end{tabular}

Data are means \pm SD or $\mathrm{n}(\%)$ unless otherwise stated. Percentages may not add up to 100 because of rounding.

*Eligibility criteria: age $\geq 18$ years, A1c 5.7-6.9\% OR fasting plasma glucose 5.6-6.9 mmol/1 (100-125 mg/dl), BMI $\geq 24 \mathrm{~kg} / \mathrm{m} 2$, and systolic/ diastolic blood pressure $<180 / 105 \mathrm{mmHg}$. 